\title{
Un discours mis en image : Paris à travers les Guides Joanne - Guides bleus (1863 à 2010). Une approche exploratoire et diachronique de l'espace touristique
}

Laurie Lepan et Philippe Duhamel

\section{OpenEdition}

\section{Journals}

Édition électronique

URL : http://journals.openedition.org/tourisme/231

DOI : 10.4000/tourisme.231

ISSN : 2492-7503

Éditeur

Éditions touristiques européennes

Édition imprimée

Date de publication : 1 décembre 2012

Pagination : 6-22

ISSN : 2109-5671

Référence électronique

Laurie Lepan et Philippe Duhamel, « Un discours mis en image : Paris à travers les Guides Joanne Guides bleus (1863 à 2010). Une approche exploratoire et diachronique de l'espace touristique », Mondes du Tourisme [En ligne], 6 | 2012, mis en ligne le 30 septembre 2015, consulté le 03 mai 2019 URL : http://journals.openedition.org/tourisme/231 ; DOI : 10.4000/tourisme.231

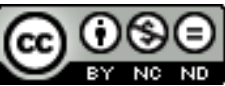

Mondes du tourisme est mis à disposition selon les termes de la licence Creative Commons Attribution - Pas d'Utilisation Commerciale - Pas de Modification 4.0 International. 


\section{Un discours mis en image : Paris à travers les Guides Joanne - Guides bleus (1863 à 2010). Une approche exploratoire et diachronique de l'espace touristique}

LAURIE LEPAN [laurie.lepan@univ-angers.fr] Doctorante, PRES LUNAM Université, UMR CNRS 6590 ESO, Université d'Angers

Phulupe Duhamel [philippe.duhamel@univ-angers.fr] Professeur des universités, PRES LUNAM, UMR CNRS 6590 ESO, Université d'Angers, UFR ITBS

Résumé. L'enjeu de cet article est d'identifier les logiques spatiales de la description d'une destination touristique d'importance: Paris. Entre 1863 et 2010, grâce au dépouillement des Guides Joanne-Guides bleus, il est possible de saisir la dynamique spatiale d'un discours, la densité des descriptions sur l'espace concerné, les logiques de hiérarchies, les permanences comme les nouveautés sur les lieux et l'espace public abordés.Ce travail est une première étape (il est réellement exploratoire) et nous interroge sur l'espace touristique décrit, ainsi que sur son évolution. II mobilise un type de document particulier : le guide de voyage. Ce dernier soulève un certain nombre de questions et problèmes relativement à l'information contenue, même si tout le monde s'accorde aujourd'hui sur son intérêt scientifique.

Abstract. The aim of this article is to identify spatial logics of a major touristic destination: Paris. Between 1863 and 2010, thanks to the analyze of travel guides (Guides Joanne-Guides bleus), it is possible to capture the spatial dynamics, the density of the description on this space, the logical hierarchy, the permanence and novelty on places and public space. This work is a first step (it really is exploratory) and asks about the touristic space and its evolution. It uses a particular tool, the travel guide, and raises some questions and problems about information even if everyone agrees today with scientific interest of such a tool. 
D

epuis une trentaine

d'années, la question

de l'usage des guides

touristiques à des fins scientifiques

a été tranchée. De la posture bien connue de Roland Barthes à celle de Daniel Nordman, un changement de "paradigme" s'opéra dans les années 1980 : "Roland Barthes dans Mythologies (1975) estimait que le Guide Bleu réduisait 'la géographie à la description d'un monde monumental et inhabité'. Dans cette optique, les Guides bleus ne renvoyaient qu'à une forme de tourisme bourgeois et à des pratiques de la culture de masse. L'article de Daniel Nordman sur les Guides Joanne (1986) dans Les lieux de mémoire, dirigés par Pierre Nora, marque un tournant dans l'historiographie: Daniel Nordman prend les guides au sérieux, les considère comme des sources, retrace leur genèse, et étudie le plan des ouvrages en rapport avec des visions du territoire national" (Cohen et Toulier, 2011).

Dès lors, les publications mobilisant cette source se sont multipliées $^{(1)}$ et, en France, ce nouveau statut scientifique des guides touristiques doit beaucoup au colloque tenu à l'université Paris-Diderot en 1997, dont les actes débouchèrent sur la réalisation d'un livre au succès commercial inédit mais révélateur de l'intérêt porté à ce type d'ouvrages: Les Guides imprimés du XVI au XXe siècle. Villes, paysages, voyages (Chabaud, Cohen, Coquery et Penez, 2000).

\section{INTRODUCTION}

LE GUIDE TOURISTIQUE : VISION DU MONDE VERSUS RÉALITÉ DU TERRITOIRE

L'utilisation des guides permet de mettre en avant un de leurs rôles majeurs. Selon certains auteurs, ils sont un discours sur un lieu et en proposent une représentation. Ils placent alors l'espace considéré en possible objet de consommation (Hancock, 2000) et en donnent les clés de lecture aux touristes (Chabaud, 2000). Leur mission est d'aller à l'essentiel (Gritti, 1967) et ils cherchent à emmener le lecteur aux principaux lieux touristiques. Ainsi ils délimitent l'espace fréquenté-fréquentable (Saunier, 1994). D'une certaine manière, les guides rationalisent au maximum l'itinéraire des touristes avec l'impératif de les prévenir lorsqu'ils doivent parcourir le chemin ou s'arrêter pour prendre le temps de découvrir.

Cela conduit à produire un espace fait de points, de lignes, d'itinéraires, de panoramas et de monuments (Saunier, 1994; Ozouf-Marignier, 201 I). Le lecteur est "guidé dans l'espace d'un texte qui se donne pour le reflet d'une réalité urbaine ainsi structurée [...]" (Chabaud, 2000); ou, comme le propose Paul-Laurent Assoun : "[Le guide] donne à voir la relation qu'entretient le texte des guides, qui construit 'une vision du monde', avec la réalité $d u$ territoire" (Vajda, 20I I). En fin de compte, il propose sa propre représentation de l'espace (Moret, 2000).

Cette "conduite du lecteur dans l'espace urbain" (Chabaud, 2000) ne se fait pas de n'importe quelle manière. Au contraire, les guides ont recours à des techniques pour structurer et organiser leur discours. Ce discours est multiple et se trouve associé à une organisation textuelle (Maingueneau, 1998). Étant un mélange entre le récit de voyage et l'ouvrage signalétique, le guide multiplie les approches (KerbratOrecchioni, 2004). Il est descriptif, puisqu'il donne des recommandations (hôtels, restaurants, visites) ; prescriptif, puisqu'il oriente le touriste lecteur et lui dit là où il doit ou ne doit pas aller ; critique, tout en restant le plus souvent positif ; publicitaire, car il doit valoriser l'espace et vendre ; enfin didactique, car il doit être considéré comme sérieux par ses lecteurs (Baider, 2004).

Ainsi le guide touristique construit un système de codes et de signes pour décrire et représenter les lieux (Bonin, 200I). Ce codage textuel s'établit à partir d'une grille descriptive qui permet de montrer la "plurifonctionnalité des lieux, la pluralité de leurs sens et de leurs usages" (Mondada, 2000, p. 40).

\section{L'approche du temps long des lieux : les choix d'une collection et d'une destination}

Pour accompagner le touriste dans son voyage, nombre de collections de guides touristiques existent à ce jour. Dans cette diversité, nous avons fait le choix de retenir les Guides Joanne, devenus les Guides bleus en 1919. Ce choix a été possible parce qu'il s'agit de l'une des trois 
grandes collections de guides. Son histoire riche et ancienne comme sa cohérence permettent une approche scientifique diachronique.

En 1826, Louis Hachette fonde sa librairie ; en 1852, il lance une collection au riche catalogue, la "Bibliothèque des chemins de fer", au moment même où se développe ce moyen de transport. Parallèlement, Adolphe Joanne (18131881) entame une carrière d'avocat, puis de journaliste, avant de publier des itinéraires de voyages, tel le Guide sur la Suisse, paru en 1841 (Rauch, 2000). Là se fait le lien entre les deux hommes, le premier proposant au second d'éditer ses guides. Dès leurs débuts, les guides sont importants, reconnus comme étant de qualité, et le directeur de publication est réputé (Guilcher, 2000). Une certaine élite y participe, comme Élisée Reclus (Bonin, 200 I). Les guides seront édités dans plusieurs séries : les "guides itinéraires", les "guides illustrés" et les "guides Diamant"(2) (Morlier, 2000). À la mort du fondateur, son fils Paul Joanne reprendra la collection, puis, en 1911, Marcel Monmarché. Enfin, 1919 est une étape importante puisque les Guides Joanne deviennent les Guides bleus - en lien avec la couleur de la couverture.

De cette histoire découle une forte cohérence et une certaine "continuité de la collection depuis un siècle et demi, avec seulement des mini-révolutions après quelques décennies de rééditions-reproductions" (Bonin, 200I). Un argument en faveur de l'usage des Guides Joanne-Guides bleus selon Sophie Bonin, qui indique que leur utilisation " $n$ ' $a$ de sens que dans une perspective diachronique, dans la recomposition d'un système de représentations" (ibid.).

Le choix de Paris tient à la relation privilégiée que cette ville entretient avec le tourisme, puisque certains n'hésitent pas à dire que le tourisme en ville fut inventé à Paris (Équipe Mit, 2005) sous la houlette des Anglais. Aujourd'hui, Paris est considérée comme l'une des principales destinations urbaines au monde, avec plus de 28 millions de touristes pour la commune elle-même et 44 millions pour la région Île-de-France (OTCP, 20 I0). Ainsi, Paris apparaît comme l'archétype de la destination touristique urbaine qui permet d'approcher sur plus d'un siècle l'évolution du discours et des codes de représentation d'une capitale, au cœur du processus touristique européen et mondial.

Les Guides Joanne-Guides bleus sur Paris deviennent un outil possible pour la découverte des représentations de l'espace urbain : "Marcel Roncayolo [...] souligne l'intérêt des guides de voyage pour l'écriture de l'histoire urbaine et pour l'étude de la construction des espaces urbains en Europe [car ils] offrent ainsi une lecture de la pratique historique de la ville" (Vajda, 20I I); et une telle "recherche sur les grandes collections constitue en même temps une contribution à l'étude de la construction des espaces urbains en Europe, depuis le milieu du XIX siècle jusqu'à nos jours... Par ailleurs, en privilégiant une approche patrimoniale, selon les hiérarchies qu'ils établissent pour ce qui est à voir et à faire, les guides contribuent à mettre en valeur ou à banaliser les lieux décrits et les traditions locales, contribuant ainsi à la mise en ceuvre d'une politique de protection et de conservation" (Vajda, 20 I I).

\section{Un échantillon, une méthode et un projet}

Quatre Guides Joanne-Guides bleus ont été retenus pour construire cette image du discours sur l'espace touristique parisien. Ils présentent une structuration similaire : outre des cartes, plans et photos (gravures pour le guide de 1863), la première partie présente les renseignements à connaître lors d'un séjour à Paris : arrivée dans la capitale, hébergement, moyens de transport, restaurants, services (ambassades, bureaux de poste...) ; ainsi qu'une présentation de la ville : population, climat, développement de Paris. C'est une caractéristique obligatoire qui se retrouve dans chaque guide touristique étudié, quelle que soit l'édition. Ensuite, chaque guide présente les lieux destinés aux touristes selon une organisation textuelle bien précise.

En 1863, Paris existe en tant que commune unique et le tourisme y devient une réalité sociale et économique. C'est pourquoi cette date nous a semblé convenir pour entreprendre une approche de l'espace touristique parisien. Le guide de 1863 est un inventaire exhaustif des lieux à découvrir ; ici, pas de par- 
cours mais une description hiérarchisée. Il se structure en chapitres par types d'aménagements - boulevards, quais et ponts, places - et d'équipements - églises, théâtres, musées, monuments de l'administration (prisons, tribunaux, morgues), colonnes et fontaines. Les boulevards, les quais et les ponts occupent une place majeure du guide; les premier et second chapitres leur sont totalement consacrés. En effet, ils sont des lieux importants de découverte et de déambulation, du fait des travaux menés par Haussmann et du nouvel ordonnancement urbain qu'ils incarnent. Déjà se joue l'articulation entre modernité de symboles spatiaux et construction d'un mythe et d'un imaginaire de Paris (Hancock, 2003).

L'édition de 1905 apparaît comme un guide de transition puisque la liste d'équipements et d'aménagements est complétée par des parcours et des itinéraires. Comme en 1863, les premiers sont classés par types : jardins, édifices religieux, édifices civils, musées, théâtres, terrains de sports, souterrains; et les seconds, à savoir les parcours et itinéraires, font l'objet de quinze sections distinguant les "quartiers majeurs" faits de lieux emblématiques : du Louvre à la Sorbonne en passant par le Champde-Mars. Dès le début du XX $\mathrm{XX}^{\mathrm{e}}$ siècle, le Guide Joanne n'est plus seulement l'exposition exhaustive et figée des monuments et édifices, il tisse du lien entre les lieux et propose des routes à suivre. Une découverte prescriptive se met en place ici.
En 1955, la première évolution majeure est la mise en image de la ville par la Seine. Dans les éditions précédentes, celle-ci faisait partie intégrante du paysage parisien, notamment parce que - et toujours dans ce souci d'inventaire - les guides évoquaient les quais et les ponts. Ici, le rôle de la Seine est nouveau, car elle devient le symbole de la monumentalité et se trouve associée aux origines de la capitale (Backouche, 2000). Alors, le statut de la Seine n'est plus seulement celui d'une voie navigable jalonnée de ports mais celui de symbole de la capitale en tant qu'élément structurant et représentatif de Paris. La deuxième évolution majeure est la disparition de la structure par types de lieux. Dès lors, la découverte de la ville se fait par vingt-sept parcours ponctués des atouts touristiques de la ville comme la modernité, la culture et surtout la monumentalité. Enfin, le guide propose non pas un système de classement des lieux mais les principales curiosités de la ville à travers les siècles : les arènes de Lutèce (l'époque gallo-romaine), le dôme des Invalides (le XVII siècle) ou la tour Eiffel (le XIX ${ }^{\mathrm{e}}$ siècle).

La dernière édition retenue - celle de 2010 - conforte les logiques décrites précédemment. Les parcours sont le mode de découverte de la ville, jalonnés par un ou plusieurs lieux représentatifs du Paris touristique : la monumentalité (le Louvre, les Invalides), l'imaginaire (Montmartre), la modernité (la tour Montparnasse, les Halles). Ici le rôle de la Seine s'exprime autrement, par une distinction entre rive gauche et rive droite, cette dernière occupant 358 pages, contre 237 pages pour la première. Cette séparation est ancienne mais c'est la première fois qu'elle structure le guide lui-même. Enfin, cette édition montre l'instauration d'un système de classement des lieux. Le système d'étoiles instauré en 1921 (Cohen, 2000) prend une place déterminante et structure fortement les cartes. Il met en place et construit une hiérarchie de la découverte entre les lieux exceptionnels (l'île Saint-Louis, le musée d'Orsay), très intéressants (les Tuileries, le musée du quai Branly) et intéressants (le Châtelet, la place du Tertre). La majeure partie des lieux cités dans le guide n'a pas d'étoiles.

Le rythme mi-séculaire correspond à la volonté d'avoir une approche qui éclaire régulièrement l'espace jusqu'à nos jours. Il tient aussi à la longueur du dépouillement (cf. encadré 1 ) et du traitement de l'information, qui n'autorise pas, dans le cadre d'un article exploratoire, à retenir plus de dates. Nous avons constitué une base de données à partir du dépouillement des guides et nous avons cartographié les résultats. Ils constituent la matière de la présente analyse.

Ici nous ne faisons pas d'analyses lexicales mais nous recherchons à voir l'évolution de l'espace touristique décrit par les guides au fil du temps. Ce qui est important est de savoir la quantité de mots (exprimée en caractères) consacrée à tel ou tel lieu ou équipement en les localisant 


\section{Encadré $\mid \cdot$ Méthodologie du dépouillement}

L choix a été fait de dépouiller quatre guides touristiques de la même — collection. Pour chaque lieu et rue cités dans chacun des guides, nous avons pris en compte le volume du texte (nombre de caractères) ainsi que les différents niveaux de typographie. Pour faciliter le dépouillement, chaque ligne inférieure à une demi-ligne $=0$ ligne, et chaque ligne supérieure à une demi-ligne $=1$ ligne.

Nous nommons "lieu" les édifices publics (sans vocation touristique), les musées, l'équipement urbain (statue, fontaine, colonne), les parcs et jardins et les monuments (ainsi appelés lorsque les édifices possèdent une logique touristique forte - découverte, panorama, visite) : ils seront représentés sous forme d'un point. L'espace public (que nous nommons "rue") - avenues, boulevards, quais, ponts et rues - est représenté sous forme de ligne.

Chaque année retenue a été croisée avec les autres pour identifier les éventuelles appellations différentes qui désignaient en réalité un même lieu, une même rue. Ainsi fut constituée la matrice de données à partir de laquelle nous avons réalisé nos traitements.

systématiquement. Plus qu'une lexicométrie, c'est une topométrie que nous proposons : il s'agit bien de voir ce qui est intégré au guide et ce qui ne l'est pas; ce qui l'a été et ne l'est plus; et de mesurer la hiérarchie qui s'établit entre les lieux au fil du temps.

\section{OBservation \\ D'UN ESPACE TOURISTIQUE EN FORMATION}

Une première mise à plat des lieux et des rues identifiés dans les guides permet de saisir le semis et le treillage de l'espace parisien par le tourisme. C'est une ossature de l'espace qui se donne à voir.

\section{L'ossature}

de l'espace touristique parisien

Comme le propose la typologie des lieux touristiques, Paris est une ville touristique et, à ce titre, c'est un espace investi et diverti par le tourisme (Équipe Mit, 2002 ; Stock, 2006). Tout l'espace parisien n'est pas touristique car "il y a l'insertion d'une fonction touristique dans l'espace” (ibid.). Cela signifie que le tourisme vient compléter les activités de la ville par l'apport d'une population nouvelle, de "nouveaux voyageurs" : les touristes qui passent et qui séjournent. Dès lors, le tourisme se développe, généralement, sans modification fondamentale de la structure urbaine. Même si "théoriquement toute la ville intéresse sans réelle distinction: les édifices comme les quartiers douteux, [...] progressivement se mit en place un espace distingué par les voyageurs ne privilégiant que certains pans $d u$ territoire parisien” (Duhamel, 2007).

Le dépouillement des Guides
Joanne-Guides bleus confirme largement cette analyse. En effet, on recense plus de huit cent lieux et trois cent dix-huit rues et boulevards présents sur l'ensemble de la commune. Si l'on regarde le nombre de points et de rues décrits par les guides selon les grandes étapes du développement de la ville de Paris, on aboutit au constat d'un bel équilibre aujourd'hui (cf. illustration 1). Le Paris de Charles V (cf. illustration 2) concentre 174 lieux et 91 rues, soit $34 \%$ du total; le Paris du XVIII ${ }^{\mathrm{e}}$ siècle, enserré par l'enceinte des Fermiers généraux, rassemble 180 lieux et 85 rues, soit $34 \%$ de l'échantillon, et le nouveau Paris intégré en 1860 regroupe 194 lieux et 52 rues, soit $31 \%$ du total. En revanche, les contrastes apparaissent par les différences de densité.

Trois niveaux sont identifiables. Le premier se cale sur le cœur de Paris, le "Paris historique" (Hancock, 2003) délimité par les boulevards - les Grands Boulevards au nord, le boulevard du Montparnasse au sud -, où l'on recense 265 lieux ou rues sur plus de $6 \mathrm{~km}^{2}$, soit plus de 42 "lieux/rues" par km² ; cette densité chute à 7,5 pour l'espace compris entre le Paris médiéval et celui du XVIII ${ }^{\mathrm{e}}$ siècle (265 lieux/rues) pour atteindre un peu plus de 4 avec les espaces annexés en 1860 (246 lieux/rues). Enfin, la Seine apparaît comme un axe transversal à ces ensembles avec plus de 100 lieux/rues présents à moins de 250 mètres de ses rives. 


\section{La formation}

\section{de l'espace touristique}

L'analyse de différents guides touristiques permet aussi d'avoir une visibilité sur le temps long des lieux et des rues décrits, à destination des touristes. Au fil du temps, différentes logiques se mettent en place avec une pérennité pour certaines et des changements pour d'autres ( $c f$. illustration 3).

En 1863, l'espace touristique est largement concentré dans le centre historique de Paris, dans les $1^{\text {er }}$ et $4^{\mathrm{e}}$ arrondissements, autour du Louvre, du Palais de justice et de NotreDame. Ce premier anneau s'étend aussi vers le sud au niveau du jardin et du musée du Luxembourg jusqu'au Jardin des Plantes, intégrant l'ensemble Sorbonne-Panthéon. La densité la plus forte se trouve autour de ces édifices et le long de la Seine. Quelques rues et boulevards sont cités, notamment dans le périmètre délimité auparavant par l'enceinte de Charles V, qui réapparaît spatialement à travers la présence des boulevards du Montparnasse et des Italiens. Cette concentration semble indiquer un premier anneau, ou "hypercentre", qui rassemble cent soixante-treize lieux, soit $43 \%$ du total et $36 \%$ des rues. Au-delà de cette hyper-concentration, une première extension vers l'ouest se dessine autour et à partir de l'avenue des Champs-Élysées puis vers les Invalides ; extension qui se développe dans une continuité spatiale avec l'hypercentre.

En 1905, deux évolutions apparaissent. La première est une exten- |llustration | • Lieux et rues décrits dans les guides (I 863-20 I0)

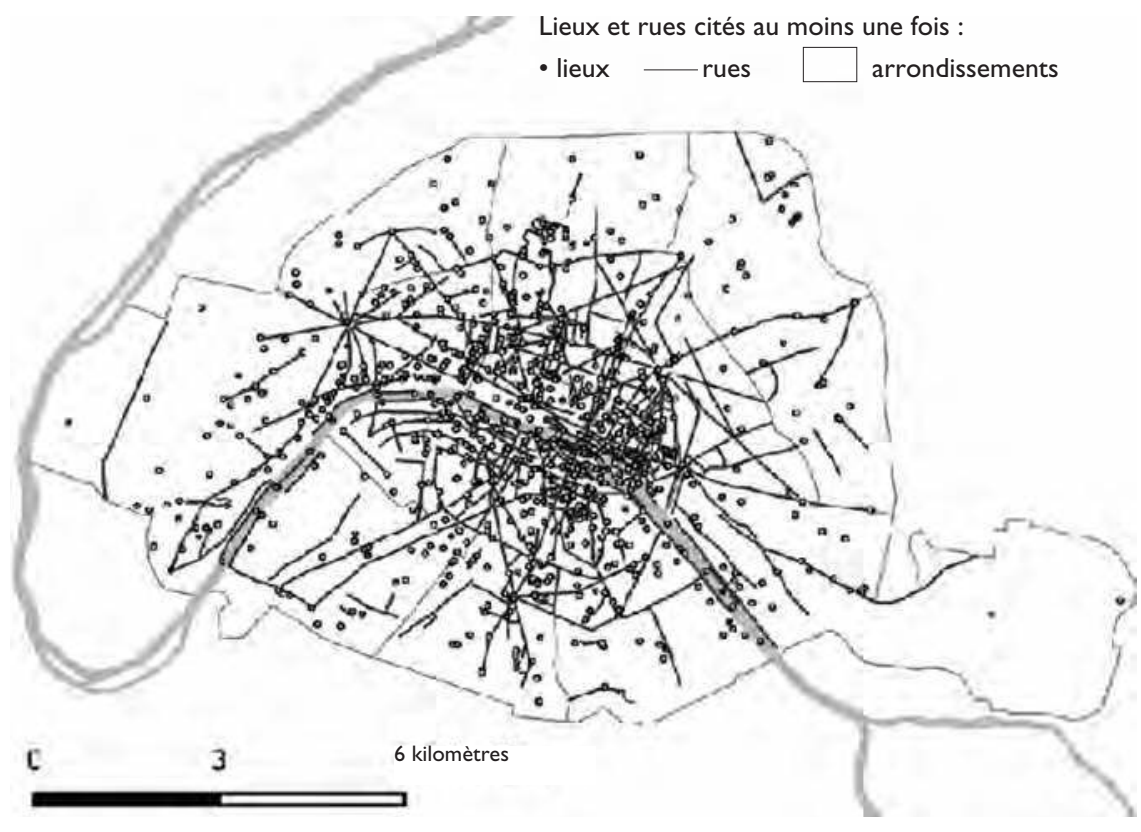

Sources: Guides Joanne et Guides bleus.

Conception-réalisation : P. Duhamel, L. Lepan, S. Giffon, université d'Angers, Eso-Angers, 201 I.

Illustration $2 \cdot$ Le Paris de Charles V

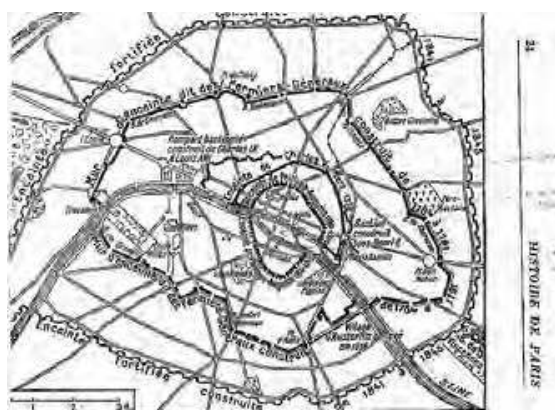

sion de la densité ; la deuxième, une extension de l'espace touristique parisien. L'hypercentre qui prend forme dès 1863 est toujours présent en 1905. Les rues et boulevards sont nettement plus décrits dans le guide, notamment les rues des FrancsBourgeois ou Vieille-du-Temple et les boulevards Saint-Germain et des Italiens. Leur nombre passe de trente-deux à quatre-vingt-dix et représente la moitié des voies décrites. Cette situation est accentuée par l'addition de nouveaux lieux aux côtés du Louvre ou de NotreDame - la Bibliothèque nationale, les Archives, le musée Carnavalet, le Palais- Royal - mais aussi par le développement du quartier de l'Opéra. 


\section{Illustration $3 \cdot$ Lieux et rues cités au moins une fois dans les guides}
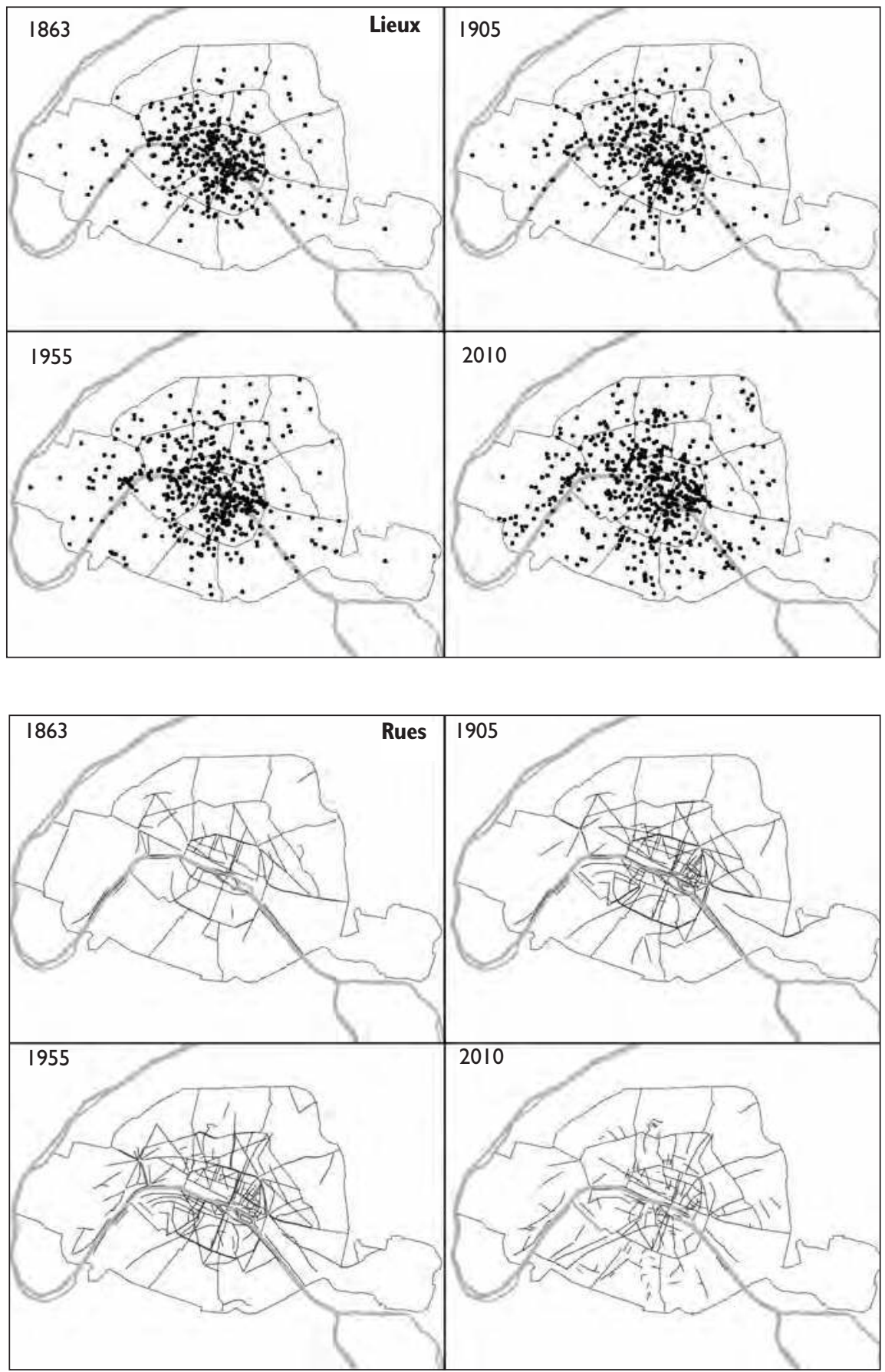

8 kilomètres

Sources : Guides Joanne et Guides bleus.

Conception-réalisation : P. Duhamel, L. Lepan, S. Giffon, université d'Angers, Eso-Angers, 201 I.
L'évolution la plus représentative en 1905 concerne l'extension de l'espace touristique parisien vers l'ouest, déjà amorcée en 1863, mais en plein développement ici. Celle-ci s'explique par la succession des Expositions universelles entre les deux dates étudiées $(1867,1878,1889$ et 1900). Elles se sont implantées sur le Champ-de-Mars - jusque-là propriété de l'armée -, sur l'esplanade des Invalides, au Trocadéro et sur les bords de Seine. Outre la présence de plusieurs dizaines de millions de visiteurs lors de ces Expositions (32 à 51 millions sur la période considérée) (Ageorges, 2006), la conservation de certains monuments comme le palais du Trocadéro, la tour Eiffel, le pont Alexandre III mais aussi les Petit et Grand Palais (Exposition de 1855) crée de nouveaux attraits pour la découverte touristique. Enfin, dans le $16^{\mathrm{e}}$ arrondissement, dix-huit lieux nouveaux sont décrits : ainsi le musée de l'Homme, le musée Galliera ou le pont d'Auteuil.

En 1955, l'hypercentre défini dès 1863 est toujours très présent mais perd de son poids relatif avec seulement $37 \%$ des lieux ( $43 \%$ en 1863) mais reste au même niveau pour les rues. Un deuxième ensemble s'étendant entre l'enceinte de Charles V et l'enceinte des Fermiers généraux concentre $37 \%$ des lieux et $38 \%$ des rues. La description de l'ouest parisien devient plus présente et prend une ampleur considérable avec la domination du noyau palais du Trocadéro - musée des Monuments français -, musée Guimet ou École militaire. Le $16^{\mathrm{e}}$ 
arrondissement gagne treize lieux et le $7^{\mathrm{e}}$ arrondissement est nettement représenté avec le musée de l'Armée, l'église Saint-Louis des Invalides ou l'hôtel des Invalides et croît de sept lieux. Ainsi émerge un chapelet continu de lieux en jonction avec l'hypercentre touristique. L'extension se poursuit également vers le nordouest, l'Arc de triomphe, le parc Monceau et le musée Nissim de Camondo. Elle se déploie aussi vers le sud de Paris au-delà du boulevard du Montparnasse avec l'Observatoire ou le cimetière du Montparnasse. Cette dynamique se fait de manière continue et, spatialement, se dessine très clairement. Mais elle fonctionne également de manière discontinue avec l'apparition de satellites - bois de Boulogne, bois de Vincennes qui occupent de plus en plus de place dans les guides touristiques. Ainsi, le premier passe de 2350 caractères en 1900 à $8800^{(3)}$ en 1955 ; le second, de 5800 en 1905 à 8500 en 1955 . De plus, les rues et les boulevards atteignent ici leur apogée avec 223 axes identifiés ( 89 en 1863 ; 179 en 1905 et 168 en 2010). À cette date, boulevards, cafés et terrasses sont ancrés dans l'imaginaire prescripteur du guide et des conduites à tenir. Parmi cet ensemble, les boulevards de la Madeleine, SaintMichel, Raspail ou la rue des Archives tirent particulièrement leur épingle du jeu avec une description comprise entre 500 et 2400 caractères.

En 2010, les "fondamentaux" sont les mêmes à une exception près : la Villette. Mais leur domination est beaucoup moins nette que dans les éditions précédentes. Il est intéressant de noter qu'en 2010, le guide s'inscrit spatialement sur l'ensemble de la ville, avec seulement quelques "vides" dans les $18^{\mathrm{e}}$ ou $19^{\mathrm{e}}$ arrondissements mais aussi dans une grande continuité spatiale entre le "centre" et l'ouest de Paris. Les rues et boulevards si présents en 1955 tendent à perdre de l'intérêt et le maillage est moins continu. Seuls les principaux (Italiens, Haussmann, Montparnasse, etc.) persistent. L'élément important de ce guide est l'apparition de lieux nouveaux au sud et plus précisément dans les $13^{\mathrm{e}}, 14^{\mathrm{e}}$ et $15^{\mathrm{e}}$ arrondissements, autour de Montparnasse, de Bercy ou du quartier chinois : on passe de 46 à 75 lieux décrits. Ce changement est parfaitement illustré par la revalorisation des quartiers de Bercy, de la cour Saint-Émilion et le développement de la Zac Masséna, l'ensemble se structurant autour de la Bibliothèque FrançoisMitterrand, inaugurée en 1995. Dorénavant, l'équilibre est atteint entre les trois grandes entités du développement parisien comme énoncé préalablement.

Cette évolution de l'espace touristique parisien peut s'exprimer à travers la surface de la ville décrite dans les guides. Elle montre une logique particulière. Dès 1863, 57 \% du total est décrit dans les guides (cf. illustration 4) et l'on atteint $59 \%$ en 1905. L'évolution la plus forte se situe entre 1905 et 1955 avec une progression de $12 \%$ de l'espace parisien intégré ( $71 \%$ du total) dans le guide. En 2010, on atteint 74 \% de la superficie de Paris commentée par le guide touristique. Une telle observation tendrait à prouver que les évolutions sont moins dans la conquête d'espaces nouveaux que dans la densification d'espaces conquis.

\section{DYNAMIQUES \\ ET HIÉRARCHIE DES LIEUX}

Mais, au-delà d'un équilibre apparent dans les semis de points et les treillages de lignes comme d'une croissance relative de la surface décrite, il convient d'observer la densité et la hiérarchie des lieux et des axes commentés. Elles créent une ville touristique nettement contrastée.

\section{Un espace touristique \\ plus vaste au fil du temps : \\ densité, densification et Central Tourists District}

Tout d'abord, la distribution des lieux et des rues selon l'importance de leur description montre deux logiques historiques. En effet, pour atteindre le seuil des $25 \%$, $50 \%$ et $75 \%$ du texte total des guides, l'espace parisien montre des dynamiques contrastées ( $c f$. tableau 1). D'une part, de 1863 à 1905, le nombre de lieux rassemblant $50 \%$ du texte ne cesse de diminuer : de 32 à 21 lieux. Un processus de concentration des descriptions s'opère. Puis une amorce de diffusion s'observe en 1955 avec 25 lieux. Et cela est encore plus représentatif pour les lieux qui rassemblent un quart du texte écrit : de 10 à 4 puis 


\section{Illustration 4 •Évolution des zones couvertes par les citations des guides}

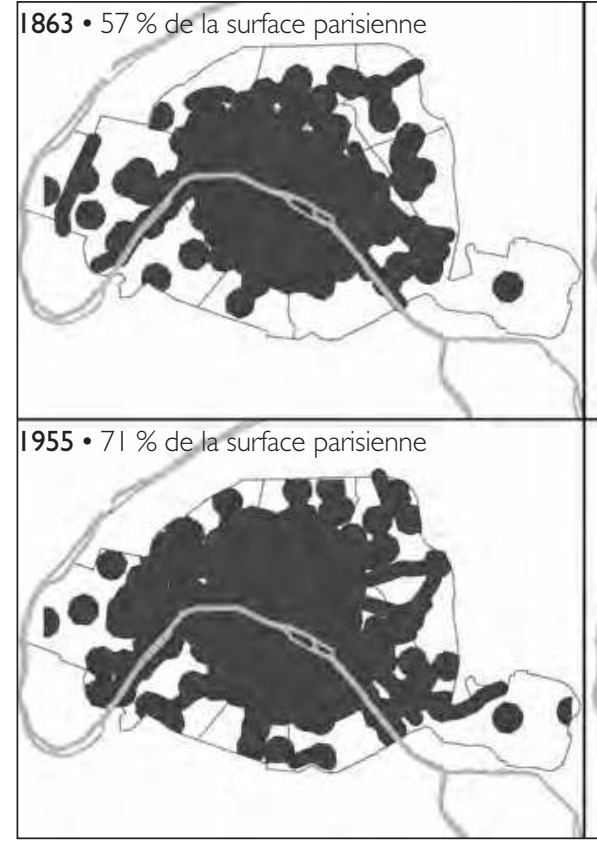

Prise en compte des lieux et des rues

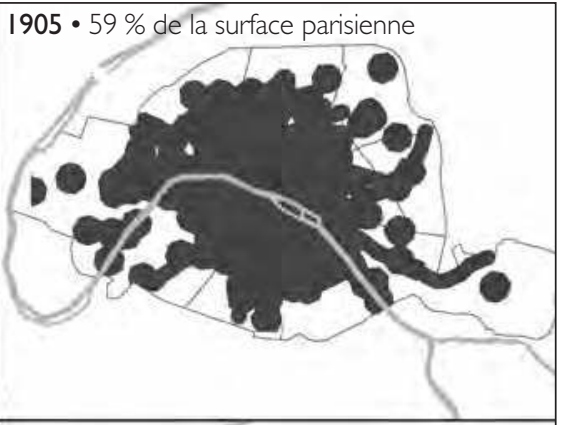

$2010 \cdot 74 \%$ de la surface parisienne

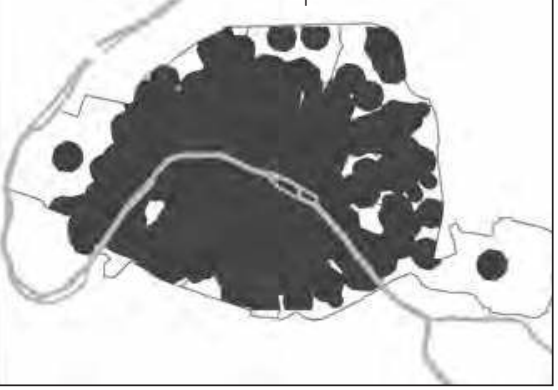

8 kilomètres

Sources : Guides Joanne et Guides bleus.

Conception-réalisation : P. Duhamel, L. Lepan, S. Giffon, université d'Angers, Eso-Angers, 20I I.

\section{Tableau | • Indice de concentration textuel des lieux}

$\begin{array}{llll}\text { Guide } & 25 \% \text { du guide } & 50 \% \text { du guide } & 75 \% \text { du guide } \\ 1863 & 10 \text { lieux } & 32 \text { lieux } & 102 \text { lieux } \\ 1905 & 4 \text { lieux } & 21 \text { lieux } & 53 \text { lieux } \\ 1955 & 3 \text { lieux } & 25 \text { lieux } & 79 \text { lieux } \\ 2010 & 7 \text { lieux } & 43 \text { lieux } & 166 \text { lieux }\end{array}$

à 3 en 1955. Le musée du Louvre est ici exemplaire de cette dynamique car il rassemble $6 \%$ du texte contenu dans le guide de 1863 , $15 \%$ en 1905 et $19 \%$ en 1955 . En 2010, la diffusion est davantage présente, elle accentue la dynamique de 1955 puisque cinquante lieux représentent $50 \%$ du texte, et qu'il faut cent soixante-six lieux pour atteindre les $75 \%$ soit trois fois plus qu'en 1905 et deux fois plus qu'en 1955. À cette date, le musée du Louvre voit son poids relatif diminuer, avec seulement $14 \%$ du texte du guide.
Ce dépouillement permet de mettre en lumière la notion de "Central Tourists District” (CTD) (Burtenshaw, Bateman et Ashworth, 1991 ; Duhamel et Knafou, 2007) : cette notion permet d'identifier un espace touristique fort délimitable - " au nord avec le boulevard des Italiens, l'Opéra et les Grands Magasins ; à l'ouest, l'Arc de triomphe et le Trocadéro jalonnent la limite; sur la rive gauche, l'espace touristique comporte quelques points forts comme la tour Eiffel, les musées du quai Branly et d'Orsay et l'Institut du monde arabe, et pénètre dans les terres jusqu'au jardin $d u$ Luxembourg, voire la tour Montparnasse, se réduisant ensuite vers l'est à un liseré” (ibid.) - où se concentreraient les sites, les monuments, les musées, une partie des hébergements mais surtout où les touristes seraient visibles dans les rues.

Le dépouillement des guides touristiques permet de corroborer l'hypothèse d'un “CTD” puisqu'un espace très décrit (supérieur à 5000 caractères) apparaît nettement et se distingue du reste du territoire ( $c f$. illustrations 5 et 6). Il est un peu décalé vers l'ouest par rapport à l'enceinte de Charles V, et montre dès 1863 des satellites inattendus comme les bois de Vincennes et de Boulogne, le cimetière du PèreLachaise et l'Arc de triomphe. Il s'étend progressivement : de 1300 hectares en 1863 à 1600 en 1905 , 2400 en 1955 et 2800 en 2010. Aujourd'hui, il couvre la quasi-totalité du Paris médiéval et au-delà, 
vers l'Ouest parisien avec les $7^{\mathrm{e}}$ et $8^{\mathrm{e}}$ arrondissements, très fortement décrits dans les guides contemporains. Le $16^{e}$ arrondissement, à partir de la Seine, progresse dans la description des guides.

Le CTD textuel s'étend au sud dès 1905 et des satellites apparaissent dès cette époque aux confins du $14^{\circ}$ arrondissement avec la place DenfertRochereau et les Catacombes. L'Est parisien, très à l'écart encore en 1905 , est plus décrit en 1955 et particulièrement investi par le texte de 2010 (cf. illustration 7). Ce premier travail exploratoire permet donc de saisir autrement le Central Tourists District parisien et permettrait de l'affiner par la confrontation avec celui que proposent Philippe Duhamel et Rémy Knafou (2007) (cf. illustration 8).
Illustration 5 - Rues citées dans les guides : évolution du nombre de caractères

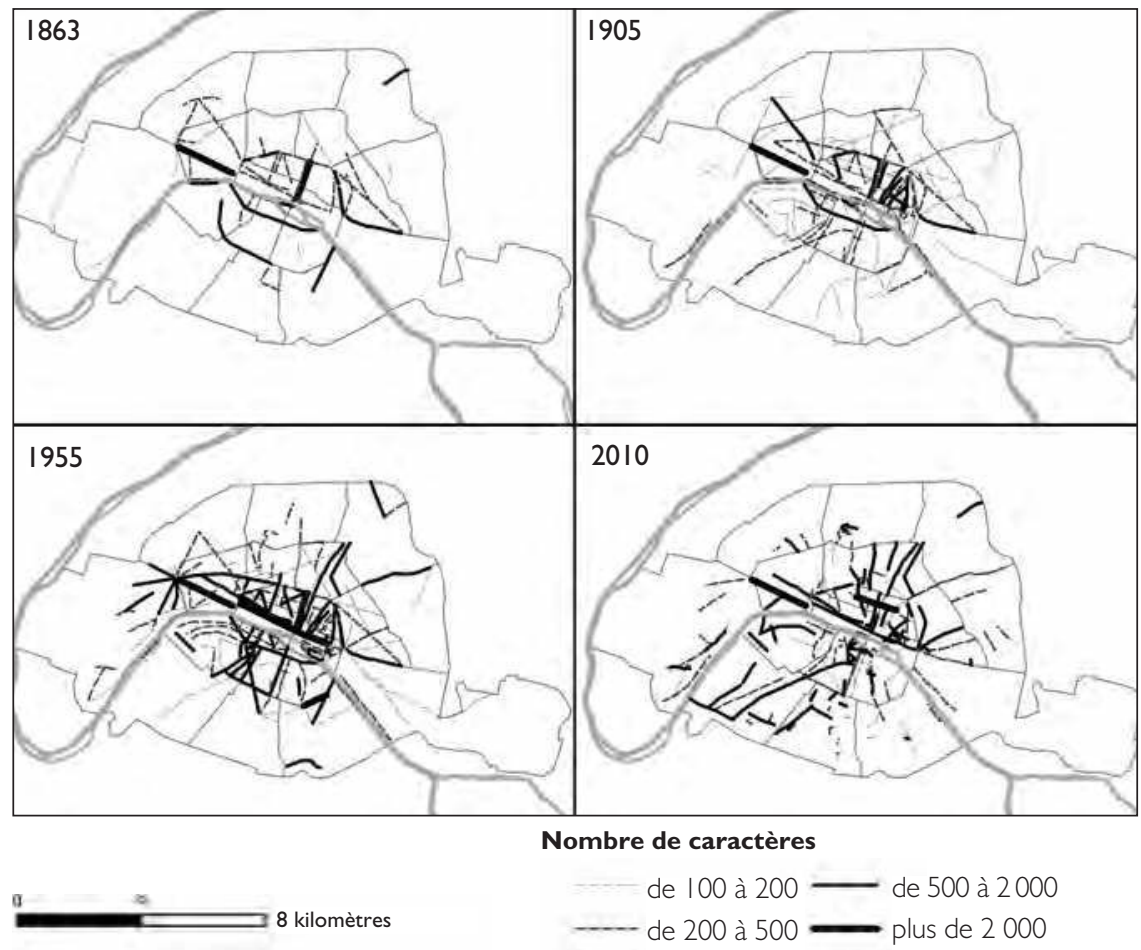

Illustration 6 •Évolution de la densité de lieux cités dans les guides

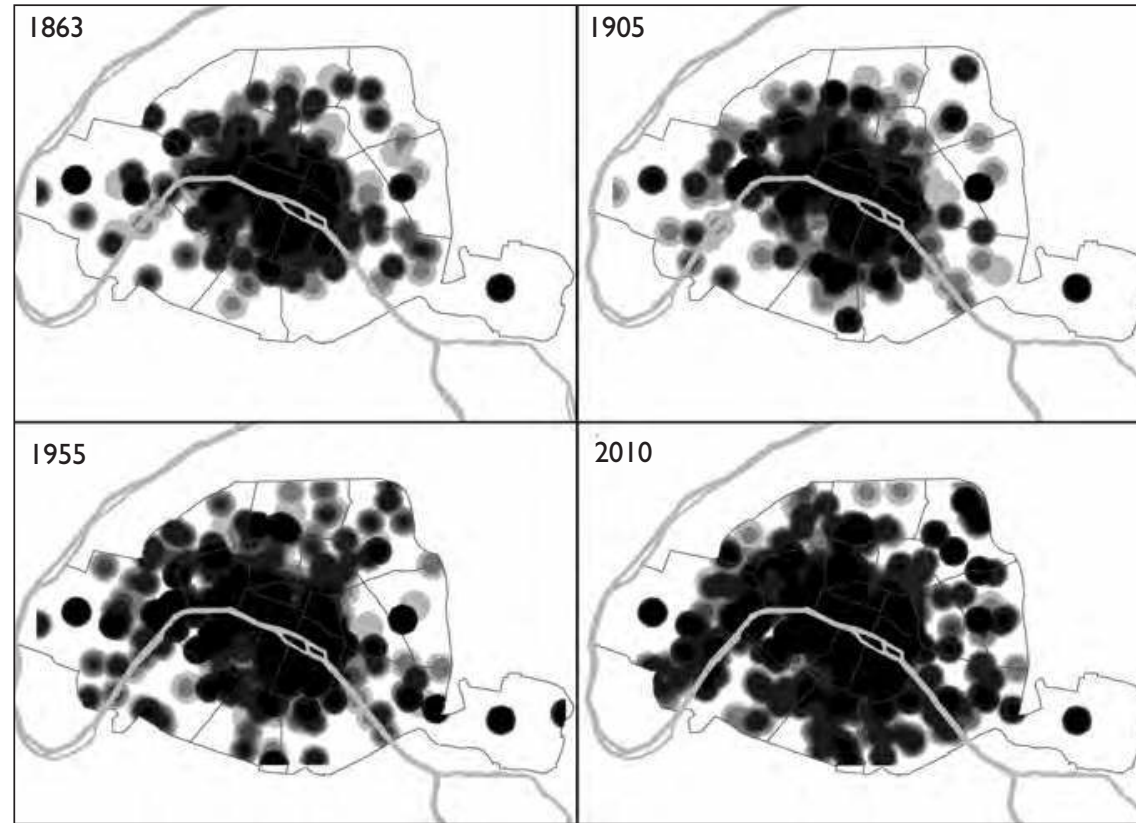

Nombre de caractères

$\square 0 \square 0-100 \square 100-500 \square 500-1000 \square$ । 000 - $5000 \square$ Plus de 5000
La méthode utilisée pour l'interpolation des données est un calcul de densité qui répartit la quantité d'observations ponctuelles sur une unité de surface pour créer un raster continu.

Sources: Guides Joanne et Guides bleus. Conception-réalisation : P. Duhamel, L. Lepan, S. Giffon, université d'Angers, Eso-Angers, 2011 
Illustration 7 - Le CTD contemporain (et son évolution historique)

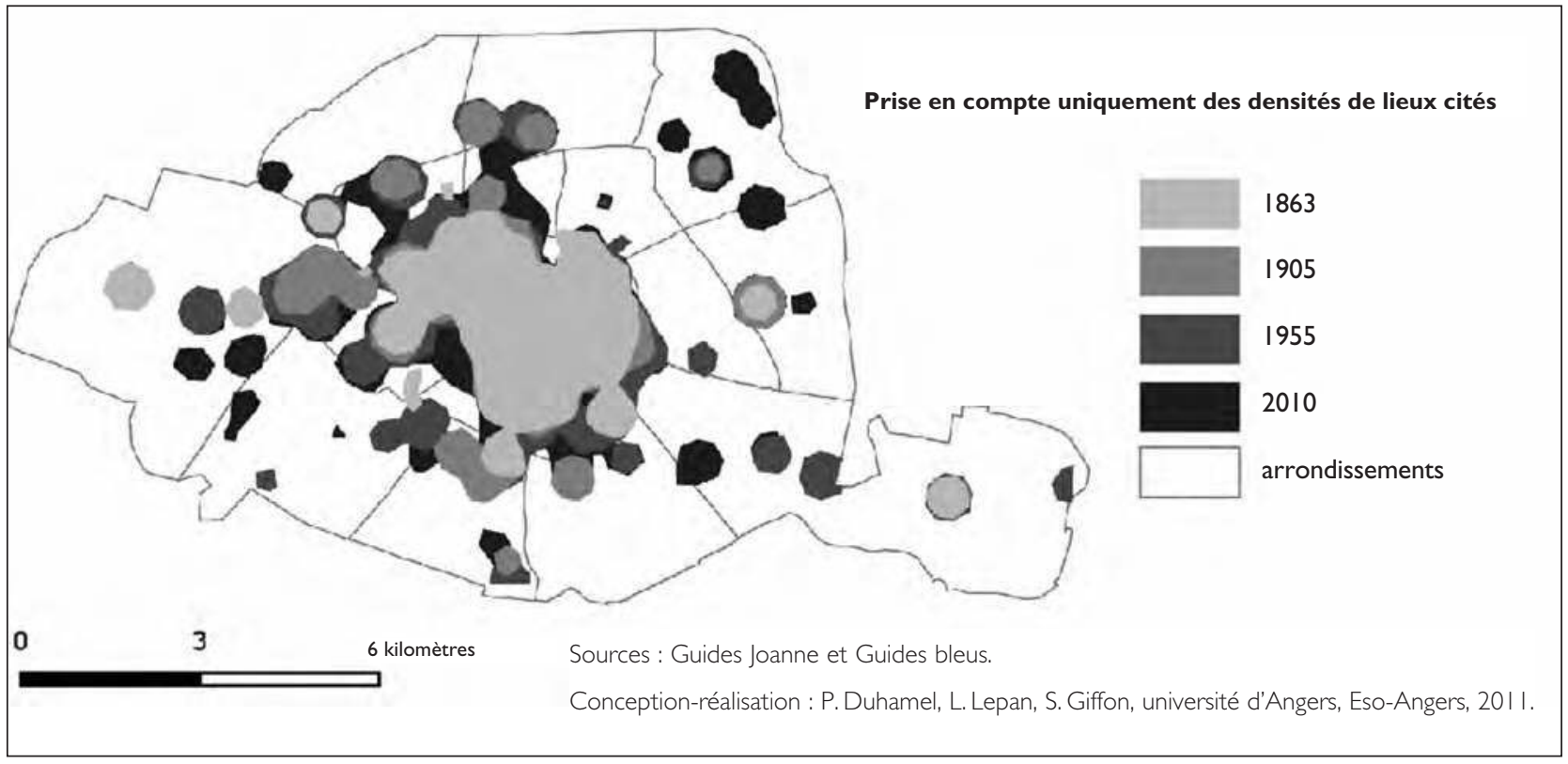

Illustration 8 - Le CTD proposé par Philippe Duhamel et Rémy Knafou

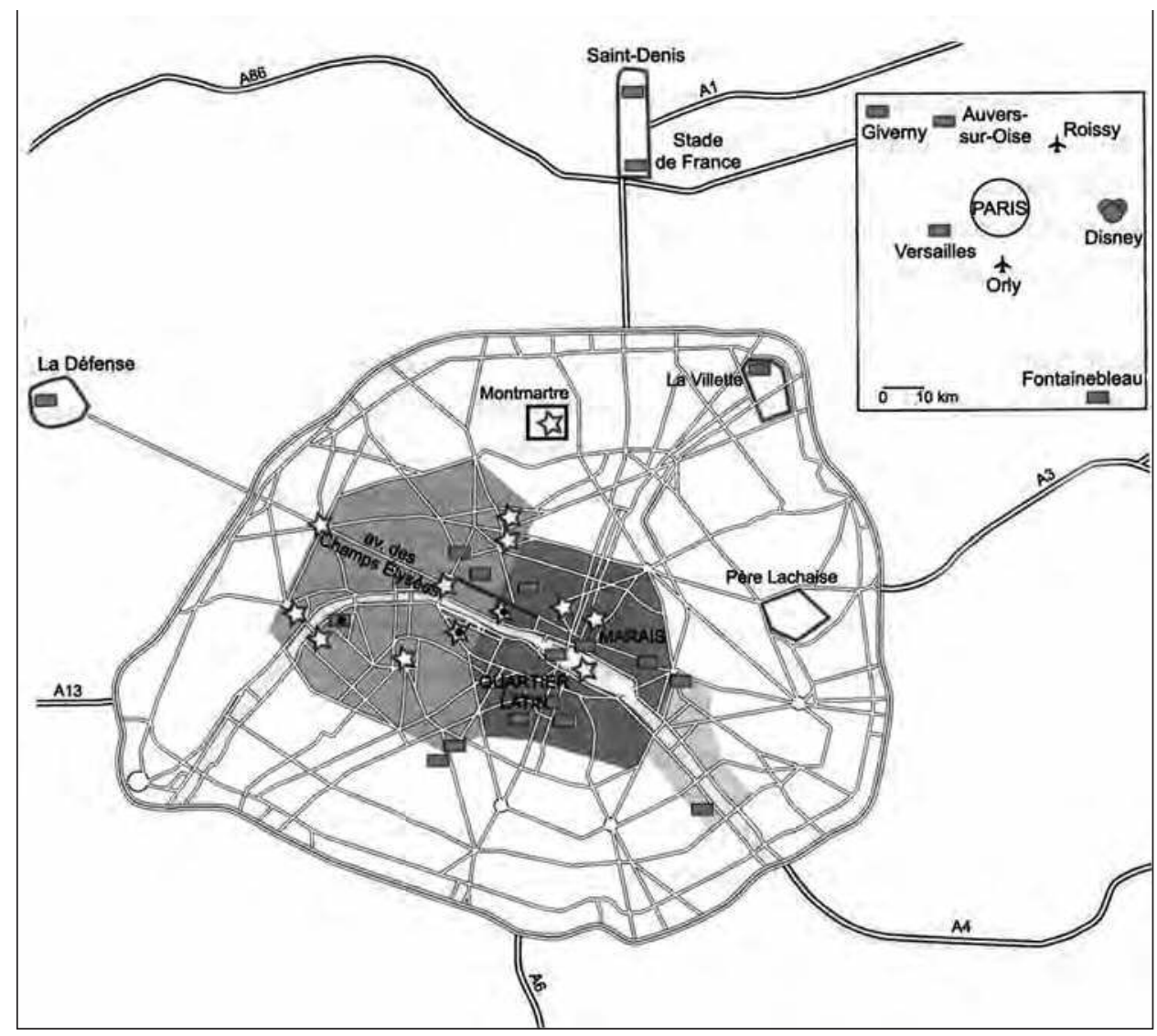

Source:

Philippe DUHAMEL et Rémy KNAFOU, "Le tourisme dans la centralité parisienne", dans Thérèse SAINT-JULIEN et

Renaud LE Golx (dir.), La

Métropole parisienne : centralités, inégalités, proximités, Belin, 2007, pp. 39-64. 


\section{L'effet de la nouveauté}

Cette densification de l'espace touristique proposée par les guides s'explique aussi par l'apparition de rues et lieux nouveaux à chaque époque (cf. illustration 9). En 1905, on en recense cent trente-cinq, puis cent vingt-huit en 1955 et deux cent quarante-sept en 2010, ce qui constitue une part toujours importante dans l'échantillon total de chaque guide (respectivement $24 \%, 19 \%$ et $35 \%$ ). La deuxième information intéressante est l'expression spatiale de la nouveauté. Elle s'inscrit dans une double logique. Tout d'abord, elle redensifie le centre puisque de nouveaux lieux surgissent dans le Marais, en 1905 et 2010 principalement. Ce processus se retrouve aussi (pour les trois guides) vers la Madeleine et l'Opéra. Ensuite, elle appuie notre hypothèse d'expansion du cœur de l'espace touristique parisien. Ce phénomène se perçoit dès le guide de 1905 pour les nouveaux arrondissements parisiens et l'expansion se poursuit avec le guide de 1955 vers l'ouest (le $16^{\mathrm{e}}$ arrondissement) comme le nord $\left(17^{\mathrm{e}}\right.$ et $18^{\mathrm{e}}$ arrondissements). Montmartre est ici un bel exemple.

En 1863, la basilique du SacréCœur n'existe pas, puis elle est décrite assez longuement en 1905 - alors qu'elle ne sera définitivement achevée qu'en 1917. Elle est largement abordée dans les guides suivants. Outre la basilique, les places et rues qui l'entourent ne sont décrites que très tard -1955 pour la place. Seule la Butte est présente tout au long de l'époque étudiée,

Illustration 9 • Les lieux nouveaux à chaque période

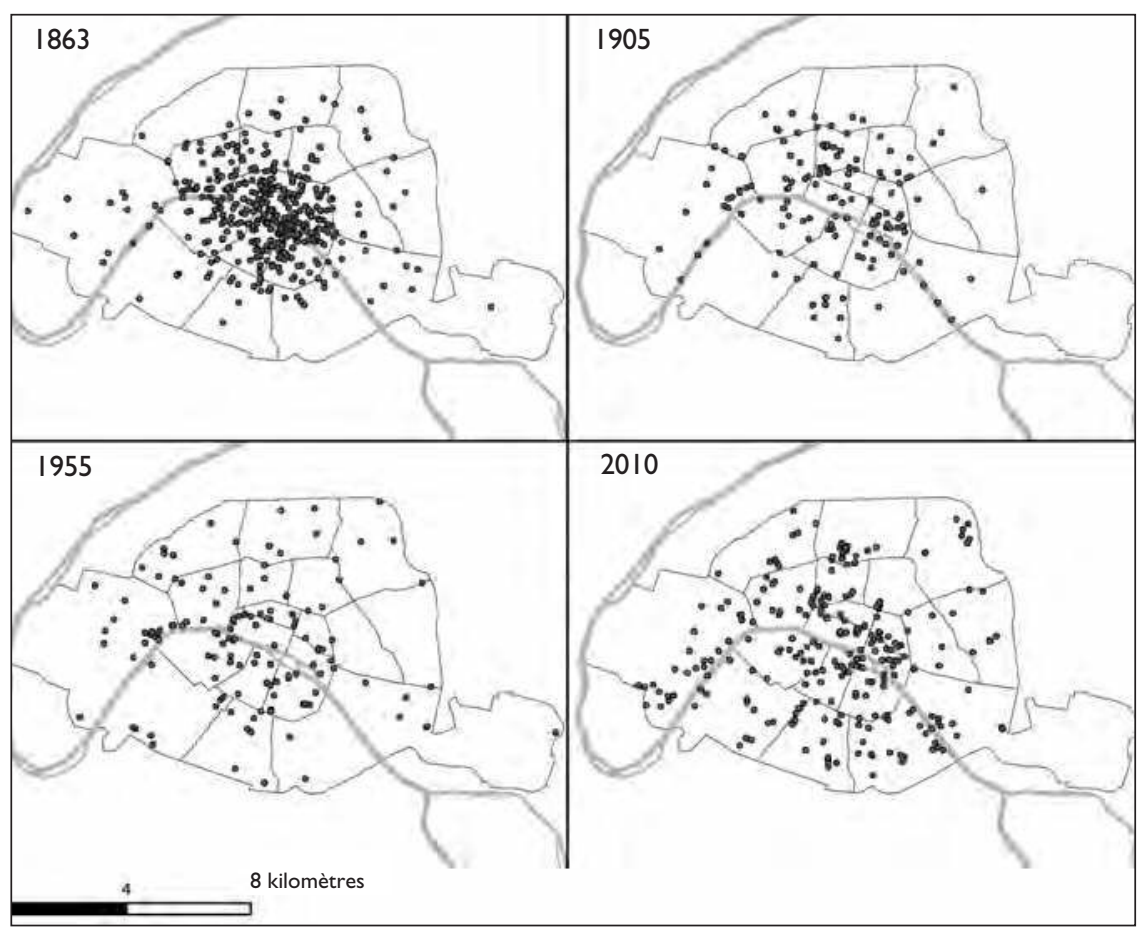

Sources: Guides Joanne et Guides bleus.

Conception-réalisation : P. Duhamel, L. Lepan, S. Giffon, université d'Angers, Eso-Angers, 201 I.

mais elle passe d'une ancienne carrière de gypse qui offre un panorama intéressant à un site de détente avec un parc et l'un des sites panoramiques majeurs de Paris. En 2010, la nouveauté investit tout autant les quartiers périphériques (avec plus de cent lieux nouveaux) que les arrondissements anciens (plus de quarante lieux dans l'enceinte de Charles V et plus de soixante lieux pour l'ancienne enceinte des Fermiers généraux).

\section{“Top 20" sur le temps long}

Les classements atténuent ce constat de diffusion à l'échelle des arrondissements comme des monu- ments eux-mêmes. Si nous retenons les vingt premiers lieux décrits par les guides depuis 1863, et que nous les associons à leur arrondissement, des recompositions apparaissent teintées d'inertie ( $c f$. illustration 10).

Tout d'abord, le $1^{\text {er }}$ arrondissement est le plus représenté jusqu'en 1955 , avec six lieux en 1863 , six en 1905 pour perdre de son influence en 1955 et être largement détrôné en 2010. En 1863, le $6^{\circledR}$ arrondissement est aussi représenté avec quatre lieux. La logique spatiale de 1863 est une concentration dans le "Paris historique". Cet état n'évoluera pas réellement en 1905 et ces deux arrondissements conserveront le 
Illustration 10 - Les vingt lieux les plus décrits, selon leur localisation dans les arrondissements

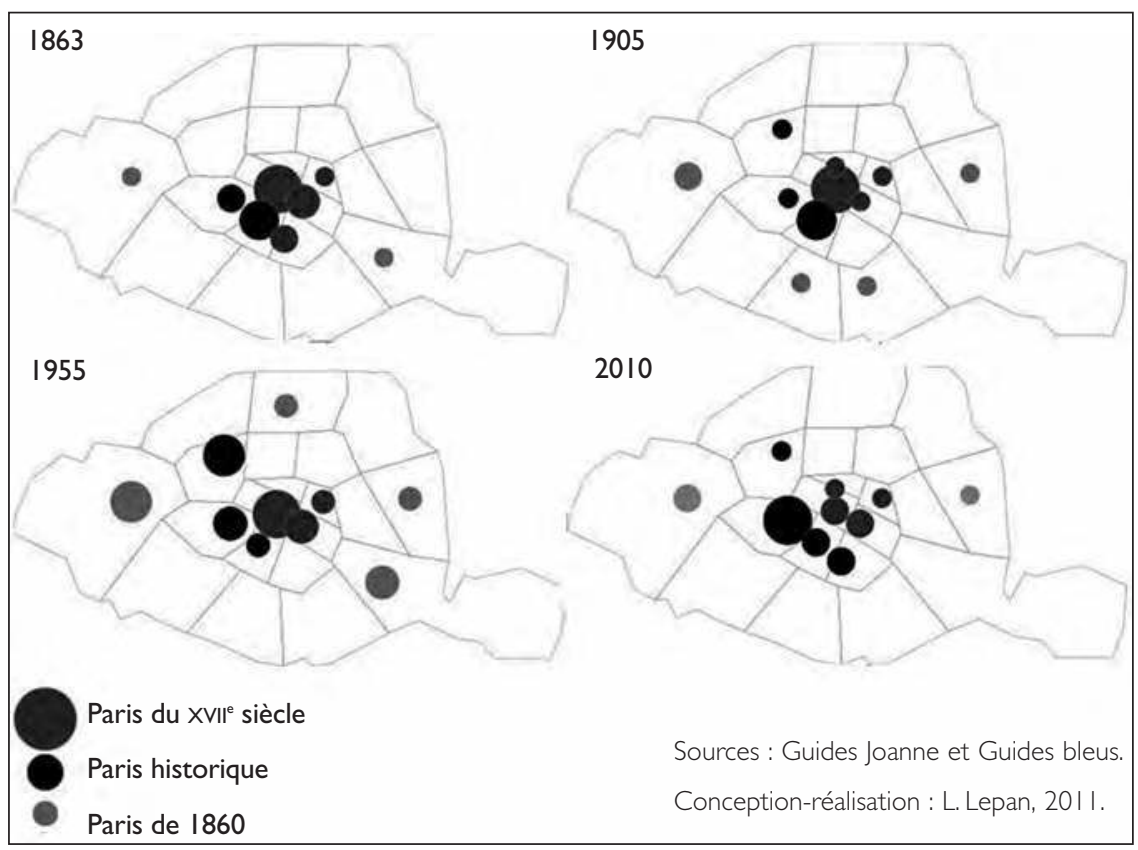

monopole du nombre de lieux les plus décrits. À partir de 1955, s'est opéré un basculement vers l'ouest de Paris : les $7^{\mathrm{e}}$, $8^{\mathrm{e}}$ et $16^{\mathrm{e}}$ arrondissements en tirent tout le profit aux dépens des $1^{\text {er }}$ et $6^{\mathrm{e}}$ arrondissements. En 2010, deux situations s'observent. Tout d'abord, le premier arrondissement perd sa domination au profit $\mathrm{du} 7^{\mathrm{e}}$ arrondissement (six lieux), il y a un déplacement du cœur du "CTD" en termes de monuments les plus commentés. Mais cette recomposition s'inscrit dans un contexte où le "top 20" ne concerne seulement que huit à onze arrondissements. L'apparente dynamique des descriptions cache une certaine inertie. Et, au-delà des volontés du guide ou des tendances contemporaines à rendre touristiques de nouveaux espaces ou des lieux différents, les classements des monuments euxmêmes distinguent les mêmes lieux (cf. tableau 2).

Le classement des lieux les plus présentés dans chacun des guides montre une variation avec l'entrée de nouveaux lieux (six à chaque période), la disparition d'autres (presque autant). Seuls dix-neuf lieux sont présents au moins deux fois. Parmi les vedettes : Notre-Dame, le palais du Louvre et le musée du Louvre sont dans le "top 20" des quatre guides, occupant une place évolutive mais d'importance. NotreDame fluctue de la septième position en 1863 , à la sixième en 1905 , puis à la septième en 1955 et enfin la neuvième en 2010. La variation est relativement faible comparée au palais du Louvre, respectivement $10^{\mathrm{e}}, 17^{\mathrm{e}}, 6^{\mathrm{e}}$ et $10^{\mathrm{e}}$. Enfin, le musée du Louvre est le lieu touristique par excellence des guides car il a conservé la première place dans les descriptions de 1863 à 2010.

Les autres lieux du classement font une apparition ponctuelle dans les guides. Cette remarque montre que le choix des lieux dignes d'intérêt n'est pas figé dans le temps et qu'audelà de quelques lieux, une dynamique d'évolution est clairement en place au sein de l'espace touristique parisien. Ainsi en est-il de l'Administration générale de l'Assistance publique en 1863, de la manufacture impériale des Gobelins, seulement présente en 1905, ou de l'église Saint-GervaisSaint-Protais en 1955. De plus, on voit l'effet de nouveauté qui contribue à faire évoluer les classements et le “CTD”, comme pour le musée Carnavalet (créé en 1890) apparu dans le guide 1905 (10 place), devenu le $2^{\mathrm{e}}$ lieu décrit en 1955 et le $4^{\text {e }}$ en 2010. À cette date, le Centre Pompidou et le musée d'Orsay, nouveaux lieux, occupent une place phare dans le descriptif de l'espace touristique parisien en densifiant le centre historique autour du Marais pour l'un, du Louvre pour l'autre.

Cette évolution est également présente dans la thématique/fonction dominante des lieux. En 1863, ce sont les édifices publics (neuf lieux) qui dominent, c'est-à-dire des lieux non touristiques tels que l'Hôtel de Ville ou l'École des beaux-arts ; suivent les parcs et jardins (cinq représentations). En 1905 , les premiers sont toujours autant présents - les Archives natio- 
nales - mais ce sont les musées qui occupent huit places. En 1955, les édifices publics diminuent dans le classement (six lieux) au profit des musées, encore un peu plus présents (neuf fois). L'espace public réapparaît un peu avec le cas de l'avenue des Champs-Élysées. Enfin, en 2010, ce sont les musées qui sont le plus représentés dans le classement (dix lieux). L'espace public, boulevards, terrasses, cafés - qui furent un symbole majeur du Paris touristique de la fin du XIX ${ }^{\mathrm{e}}$ siècle et du début du XX siècle -, n'a plus une représentativité forte.

\section{LA VIE DES LIEUX}

Enfin, le dépouillement de guides touristiques permet d'identifier autrement la dynamique des lieux touristiques décrits, montrant la stabilité de l'espace, ses renouvellements comme son mouvement chaotique. Pour cela, nous avons reconstitué l'itinéraire des lieux décrits dans les quatre guides et observé la dynamique de leur apparition et disparition. La lecture du tableau synthétique reconstitué ( $c f$. tableau 3 ) permet d'identifier quelques grandes tendances.

\section{Des lieux pérennes}

Le premier itinéraire distingue $46 \%$ des lieux dont la présence est continue dans les guides. Ils se divisent en deux sous-ensembles.

D'une part, des lieux où le sens et l'usage ne sont pas renouvelés. L'exemple le plus intéressant ici se trouve être le Louvre. Notons tout de même une évolution puisque le palais, contrairement au musée, voit sa domination diminuer. Cette pérennité est aussi dans l'usage même du lieu. Le palais est décrit comme un site touristique à découvrir et à regarder pour son archi-

\section{Tableau 2 - Le classement des monuments parisiens selon l'importance de leur description (en nombre de caractères)}

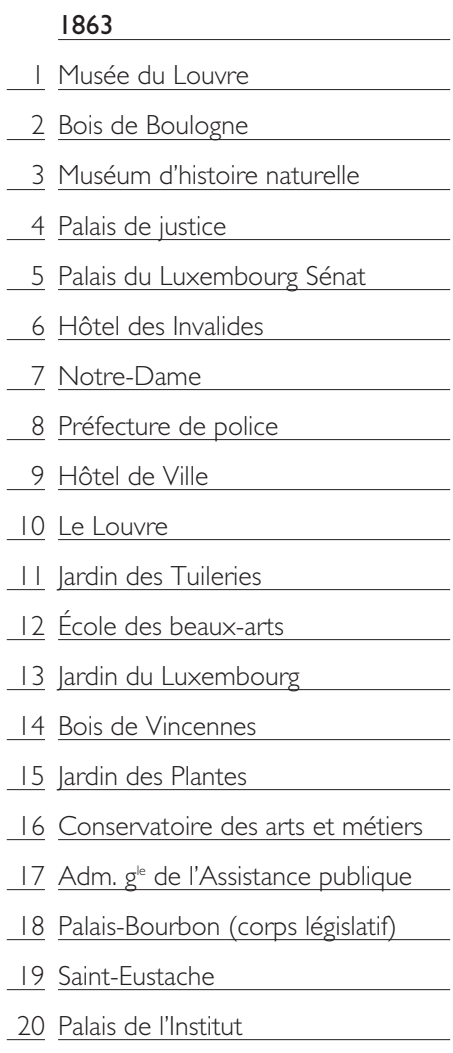

1905

Musée du Louvre
Musée Guimet
Cimetière du Père-Lachaise
Bibliothèque nationale
Musée du Luxembourg
Notre-Dame
Archives nationales
Musée des Monuments français
Musée des Beaux-Arts de la ville de Paris
Musée Carnavalet
Hôtel de Ville
École des beaux-arts
Cimetière du Montparnasse
Musée des Médailles et des Monnaies
Palais-Royal
Lardin des Tuileries
Louvre (palais)
Saint-Germain-des-Prés
Musée de l'Armée
Man. imp. des Gobelins et de la Sav.

1955

Musée du Louvre
Musée Carnavalet
Cimetière du Père-Lachaise
Musée des Monuments français
Musée Guimet
Louvre (palais)
Notre-Dame
Musée des Arts décoratifs
Musée de l'Armée
Musée des Beaux-Arts de laville de Paris
Musée de l'Outre-Mer
Musée du Jeu de paume
Bois de Boulogne
$\underline{\text { Saint-Louis des Invalides }}$
Palais-Royal
Cimetière de Montmartre
Bois de Vincennes
Saint-Sulpice
Champs-Élysées
Saint-Gervais-Saint-Protais

\begin{tabular}{l}
2010 \\
Musée du Louvre \\
Musée d'Orsay \\
\hline Musée de l'Armée \\
Centre Georges-Pompidou \\
Musée Carnavalet \\
Musée Rodin \\
Musée des Beaux-Arts de laville de Paris \\
Musée du quai Branly \\
Notre-Dame \\
Louvre (palais) \\
Hôtel de Cluny \\
Cimetière du Père-Lachaise \\
$\underline{\text { Hôtel des Invalides }}$ \\
$\underline{\text { Saint-Germain-des-Prés }}$ \\
Bois de Boulogne \\
\hline Musée Guimet \\
$\underline{\text { Saint-Sulpice }}$ \\
Opéra \\
Saint-Louis des Invalides \\
Museum d'histoire naturelle \\
\hline
\end{tabular}


Tableau 3 Présence ou absence des lieux et rues touristiques à Paris entre 1863 et 2010

\begin{tabular}{|c|c|c|c|c|c|c|}
\hline $\begin{array}{r}\text { Lieux et rue } \\
\text { apparus }\end{array}$ & Présence ou absence & $\begin{array}{l}\text { Nombre de } \\
\text { lieux ou rues }\end{array}$ & 1863 & 1905 & 1955 & 2010 \\
\hline \multirow{7}{*}{$\begin{array}{r}\text { En } 1863 \\
(449 \text { lieux })\end{array}$} & Toujours présents dans les guides & 177 & * & * & * & * \\
\hline & \multirow{3}{*}{ Absent une fois seulement } & 103 & * & * & * & - \\
\hline & & 24 & * & - & * & * \\
\hline & & 7 & * & * & - & * \\
\hline & \multirow[t]{2}{*}{ Absents deux fois } & 20 & * & - & - & * \\
\hline & & 22 & * & - & * & - \\
\hline & Absents trois fois & 96 & * & - & - & - \\
\hline \multirow{3}{*}{$\begin{array}{r}\text { En I905 } \\
(173 \text { lieux })\end{array}$} & Absents une fois & 76 & - & * & * & * \\
\hline & Absents deux fois & 11 & - & * & - & * \\
\hline & Absents trois fois & 86 & - & * & - & - \\
\hline En 1955 & & 69 & - & - & * & * \\
\hline En20I0 & & 315 & - & - & - & * \\
\hline
\end{tabular}

tecture, ses différentes statues et fresques. C'est aussi le cas du cimetière du Père-Lachaise, dont l'usage officiel est l'enterrement. Mais surtout, ce dernier concentre les sépultures de nombreuses personnalités - nationales et internationales - et, à ce titre, sa fréquentation devient une pratique touristique autour de la déambulation à la découverte de tombes de personnalités connues.

D'autre part, on identifie des lieux touristiques qui restent dignes d'intérêt durant toute la période étudiée mais un changement d'usage s'est opéré au fil du temps. Le cas de la Villette reflète cet itinéraire. En 1863, les travaux sont en cours pour la construction des abattoirs qui, une fois achevés, seront en activité jusque dans les années 1970. Ainsi, en 1905 et 1955, les abattoirs sont décrits comme les plus importants de Paris, comme un poumon économique de la capitale qu'il est possible de visiter en échange d'un pourboire donné au concierge ( $c f$.
Guide Joanne, 1905, p. 51). Si ce lieu est demeuré longtemps à usage économique et alimentaire, ce n'est plus le cas actuellement et le sens de la visite s'est largement renouvelé. Le bâtiment a été conservé pour rappeler son usage premier mais la Villette est devenue un lieu culturel fort avec la Cité des sciences et de l'industrie, la Géode, le Zénith, la Cité de la musique, mais aussi ludique avec la présence de parcs. Les Halles, autre lieu touristique important aujourd'hui pour l'activité commerciale, étaient jusqu'en 1969 le principal lieu d'approvisionnement de la capitale. L'usage commun était donc différent de la pratique touristique, le marché fonctionnant entre quatre heures et huit heures du matin.

\section{Des lieux plus éphémères}

Le deuxième itinéraire concerne des lieux dont l'intérêt touristique ne dure pas. Les logiques sont doubles. D'une part, les lieux n'ap- paraissent que le temps d'un guide (quatre-vingt-seize pour 1863, quarante-cinq pour 1905 , soixantedix-sept pour 1955), ce qui représente près de $30 \%$ de l'échantillon. Cela peut s'expliquer par l'évolution des goûts et des centres d'intérêt, comme le montrent parfaitement les descriptions des sociétés savantes, des académies ou des tribunaux qui occupaient une place assez importante en 1863 pour disparaître définitivement en 1905. D'autre part, des lieux et rues sont présents deux ou trois fois mais ont disparus en 2010. Ainsi en futil de plus de cent lieux depuis 1955, dont vingt-neuf quais et ponts et dix-huit boulevards.

Le troisième itinéraire identifie des réapparitions. Quatre-vingtquatre lieux sont dans cette situation. Il s'agit de lieux à la thématique très spécifique, par exemple les mairies d'arrondissement et les passages. Ils sont toutes et tous décrits en 1863, ont pour beaucoup 
disparu en 1905, mais sont réapparus en 1955 ou en 2010.

Ces différents itinéraires des lieux touristiques de Paris peuvent être le résultat de deux phénomènes. D'abord, le guide doit s'adapter "aux transformations urbaines, dans un souci de 'vendre le lieu décrit" (Jaumain, 2007). Ensuite, ce peut être aussi le résultat de l'évolution des goûts et des attentes des touristes lors de leur découverte de Paris ; de ce fait, le guide doit nécessairement prêter attention à ces évolutions, ne serait-ce que pour continuer à être acheté et consulté par les touristes.

\section{CONCLUSION : APPRÉHENDER \\ LA "DESCRIPTION- CONSTRUCTION" DE L'ESPACE TOURISTIQUE SUR LE TEMPS LONG}

Le choix de dépouiller les guides touristiques sur le temps long est une méthode exploratoire qui permet de saisir la logique de distinction des lieux et des axes, d'identifier le discours et de mesurer l'évolution des intérêts. Cela permet aussi de construire un panorama des espaces attrayants et une cartographie de l'espace décrit, c'està-dire l'espace prescriptif à visée touristique. Et les résultats apportent quelques éléments intéressants.

D'une part, la description touristique des lieux couvrirait dès le début (1863) une part importante de l'espace et son extension ne serait pas aussi importante sur les siècles en dépit du saut 1905-1955, qu'il conviendrait de comprendre. De même le cœur touristique de la ville (le CTD) montrerait des logiques voisines et le saut quantitatif de l'extension se situerait plutôt entre 1905 et 1955 . De ce point de vue, il y aurait là identification d'un moment important dans l'appréciation de l'espace touristique parisien - qu'il s'agirait d'appréhender et de comparer avec l'analyse plus générale du tourisme. D’autre part, l'investissement de l'espace urbain conduirait aujourd'hui à un certain équilibre dans la ville, où les quartiers périphériques de la ville historique seraient, à leur tour, des quartiers touristiques ou en passe de l'être. Ce deuxième constat laisse songeur, au-delà du texte des guides. Car il conviendrait de regarder les pratiques et de voir ce qu'il en est réellement de la fréquentation de ces lieux. Et, d'après les enquêtes existantes, les touristes repeaters iraient voir les mêmes lieux, la nouveauté n'étant pas forcément de mise (OTCP, 20 I0). Enfin, les classements montrent des basculements de polarité entre les quartiers phares, avec un certain turn-over entre les "top 20" sur cent cinquante ans. Finalement, les lieux les plus décrits sont les mêmes pour les plus importants, et une logique de renouvellement s'observe pour les autres. Cela tient-il du guide et de ses auteurs ou cela traduit-il, ou anticipe t-il, une évolution des goûts et des intérêts ?

C'est pourquoi notre approche exploratoire de l'espace décrit par les guides doit être prolongée. Tout d'abord, il faudrait systématiser le dépouillement en intégrant plus de dates et, si possible, en intégrant d'autres collections de guides français ou étrangers. Tout cela permettrait de croiser les discours et d'identifier d'éventuelles spécificités nationales dans la description, et de voir leurs évolutions. Déceler des logiques d'hier à aujourd'hui serait également un travail utile à la connaissance et à la compréhension contemporaine des pratiques touristiques, d'une part, et de la ville comme de la métropole touristique, d'autre part.

\footnotetext{
NOTES

(I) 588 occurrences de l'expression "guide touristique" sont répertoriées par Revues.org dans la rubrique "Texte des articles scientifiques". Mais le plus important est la chronologie des occurrences : première apparition en 1984, dix occurrences à partir de 1997. Depuis 2004, on est à trente et plus, pour atteindre la centaine à partir de 2008. (2) Les guides "Diamant" sont réservés à un public plus érudit.

(3) Nous avons arrondi les chiffres.
} 


\section{RÉFÉRENCES BIBLIOGRAPHIQUES}

Sylvain AgeORges, Sur les traces des Expositions universelles. Paris 1855 1937, Parigramme, 2006.

Paul-Laurent Assoun, '“L'effet Baedeker' : note psychanalytique sur la catégorie de guide de tourisme", In Situ, revue des patrimoines, $n^{\circ} \mid 5$, 2011

Isabelle BACKOUCHE, "Construction d'un genre littéraire, construction d'un espace : les guides parisiens et la Seine (XVIII'-XIXe siècles)", dans Gilles CHABAUD et al., op. cit, 2000.

Fabienne BAIDER, La Communication touristique : approches discursives de l'identité et de l'altérité, L'Harmattan, 2004.

Sophie BoNIN, "Paysages et représentations dans les guides touristiques", L'Espace géographique, $n^{\circ} 2$, tome $X X X, 2001$.

David Burtenshaw, Michael Bateman et Gregory Ashworth, The

European city, a Westem Perspective, Fulton, 1991.

Gilles Chabaud, "Les guides de Paris du XVII ${ }^{e}$ au début du XIX siècle: remarques sur une construction historique", dans Gilles CHABAUD et al., op. cit, 2000.

Gilles Chabaud, Évelyne Cohen, Natacha Coquery et Jérôme Penez (dir.), Les Guides imprimés du XVle au XXe siècle. Villes, paysages, voyages, Belin, 2000.

Évelyne COHEN, "La hiérarchie monumentale de Paris au $X X^{e}$ siècle. Les étoiles dans les guides de tourisme consacrés à Paris", dans Gilles

CHABAUD et al., op. cit, 2000.

Évelyne COHEN et Bernard TOULIER, "Les guides de tourisme, un patrimoine et un objet d'étude", In Situ, revue des patrimoines, n 15, 201 I.

Philippe Duhamel, "Patrimoine et modernité : double logique de la production et du renouvellement des villes touristiques", dans Philippe

Duhamel et Rémy KnAfou (dir.), Mondes urbains du tourisme, Belin, 2007.

Philippe Duhamel et Rémy KNAFOU, "Le tourisme dans la centralité parisienne", dans Thérèse SAINT-JULIEN et Renaud LE GoIX (dir.), La Métropole parisienne : centralités, inégalités, proximités, Belin, 2007.

Équipe Mit, Tourismes 1. Lieux communs, Belin, 2002.

Équipe Mit, Tourismes 2. Moments de lieux, Belin, 2005.

Jules GrITTI, “Les contenus culturels du guide bleu”, Communications, vol. 10, 1967.

Guide Joanne, Paris, Hachette, 1863.

Guide Joanne, Paris, Hachette, 1905.

Guide Bleu, Paris, Hachette, 1955.

Guide Bleu, Paris, Hachette, 2010
Goulven GUILCHER, "Naissance et développement du guide de voyage imprimé : du guide unique à la série, une stratégie de conquête des lecteurs?", dans Gilles CHABAUD et al., op. cit, 2000.

Claire HANCOCK, '“City of business' contre ville du plaisir : Londres et Paris dans les guides touristiques du XIX siècle", dans Gilles CHABAUD et al., op. cit, 2000.

Claire HANCOCK, Paris et Londres au XIX siècle : représentations dans les guides et récits de voyage, CNRS, 2003.

Serge JaUMAIN, “'Une grande capitale moderne' : Bruxelles dans les guides touristiques de la seconde moitié du xxe siècle", dans Philippe DUHAMEL et Rémy KNAFOU (dir.), Mondes urbains du tourisme, Belin, 2007.

Catherine Kerbrat-Orecchioni, "Suivez le guide ! Les modalités de l'invitation au voyage dans les guides touristiques : l'exemple de lîle d'Aphrodite", dans Fabienne BAIDER, La Communication touristique : approches discursives de l'identité et de l'altérité, L'Harmattan, 2004.

Dominique MaINGUeNEAU, Analyser les textes de communication, Armand Colin, 1998.

Frédéric MORET, "L'espace et le temps des guides. Représentations et déformations de l'espace urbain parisien dans les guides 1855-1900", dans Gilles CHABAUD et al., op. cit, 2000.

Lorenza MONDADA, Décrire la ville. La construction des savoirs urbains dans l'interaction et dans le texte, Anthropos, 2000.

Hélène MORLIER, Les Guides Joanne. Genèse des Guides bleus. Itinéraire bibliographique, historique et descriptif de la collection de guides de voyage ( $1840-1920)$, Les Sentiers débattus, 2007.

Daniel NordMAN, "Les Guides Joanne. Ancêtres des Guides bleus", dans Pierre NorA, Les Lieux de mémoire, tome II : La Nation, Gallimard, 1986.

OTCP, Le Tourisme à Paris, chiffres clés, 2010.

Marie-Vic Ozouf-Marignier, "Des Guides Joanne au Guide vert Michelin : points, lignes, surfaces", In Situ, revue des patrimoines, $n^{\circ} \mid 5$, 2011 .

André RAUCH, "Du Joanne au Routard : le style des guides touristiques", dans Gilles CHABAUD et al., op. cit, 2000.

Pierre-Yves SAUNIER, "Le guide touristique, un outil pour une possible histoire de l'espace : autour des guides de Lyon I800-1914",

Géographie et cultures, $n^{\circ} 13,1994$.

Mathis Sтоск (coord.), Le Tourisme, acteurs lieux et enjeux, "Belin Sup", Belin, 2006.

Joanne VAJDA, "Introduction", In Situ, revue des patrimoines, n I5, 20 I I. 